\title{
Non-thermal microwave effects on protein dynamics? An X-ray diffraction study on tetragonal lysozyme crystals
}

\section{R. Weissenborn, ${ }^{\text {a }}$ K. Diederichs, W. Welte, ${ }^{b}$ G. Maret ${ }^{a}$ and T. Gisler ${ }^{\mathrm{a} *}$}

a Universität Konstanz, Fachbereich Physik, D-78457 Konstanz, Germany, and ${ }^{\mathbf{b}}$ Universität Konstanz, Fachbereich Biologie,

D-78457 Konstanz, Germany

Correspondence e-mail:

thomas.gisler@uni-konstanz.de
$\mathrm{X}$-ray diffraction (XRD) was used to investigate the structural and dynamical effects of microwave fields on tetragonal single crystals of hen egg-white lysozyme at a resolution of $2.0 \AA$. Using a modified slab-line waveguide allows on-line XRD to be carried out while the protein crystal is exposed to well defined microwave fields. High microwave power levels mainly lead to increased, but largely recoverable, lattice defects owing to the evaporation of crystal water. At lower microwave power levels, the presence of the microwave field results in localized reproducible changes in the mean-square displacements $(B$ factors). At particular sites, it is found that the $B$ factors even decrease with increasing microwave power. Most of these effects can be explained by a comparison of the data obtained under microwave irradiation with data obtained at elevated temperature which simulate heating owing to microwave absorption by unbound crystal water. The data show no indication of large microwave-driven displacements of structural subunits in the protein that would be expected if microwaves were to be absorbed resonantly by protein vibrations. Rather, the observed changes in the atomic mean-square displacements suggest that if microwaves couple non-thermally to globular proteins at hydration levels at which they still function, their effect on protein dynamics and structure is very small.

\section{Introduction}

After more than three decades of research on the interaction of microwaves with biological matter, heating arising from the strong absorption of microwaves by the ubiquitous water has been recognized as their main effect (Polk \& Postow, 1996). On the other hand, numerous observations indicate that in addition to heating, microwave radiation might also induce non-thermal effects in narrow frequency windows that could not be explained by bulk heating. Such window effects have mainly been investigated in vivo on the cellular level where, for example, modulation of cell growth and mutation rates or enhanced expression of heat-shock proteins under the influence of microwaves has been observed (for a comprehensive review of the literature up to 1996, see Postow \& Swicord, 1996). Despite the renewed relevance of the interaction of microwaves with biological systems for the estimation of possible hazards emerging from wireless telecommunication devices, a consensus on the origin and even existence of nonthermal microwave effects has not been reached to date (Postow \& Swicord, 1996; Gos et al., 1997; Utteridge et al., 2002, 2003; Lerchl, 2003; Kundi, 2003; Goldstein et al., 2003).

Resonance absorption by low-frequency vibrations has been suggested by Fröhlich (1968) as a molecular mechanism that would allow non-thermal coupling of microwave fields to
Received 24 August 2004 Accepted 24 November 2004 
protein dynamics. Dielectric spectroscopy shows that the microwave absorption in globular proteins mainly arises from rotational relaxation of weakly bound water molecules (Harvey \& Hoekstra, 1972; Dachwitz et al., 1989) and does not show any sharp resonances indicative of long-lived vibrational states.

On the other hand, computer simulations including realistic water suggest that intramolecular vibrations are overdamped at gigahertz frequencies owing to the presence of water. These simulations make resonant coupling of microwave fields to intramolecular vibrations rather unlikely for proteins in the hydrated environment that is required for a catalytically active structure (Adair, 2002). Yet, the spectrum of diffusive anharmonic modes associated with exchange between neighbouring conformations might still extend well into the gigahertz frequency range and even below, as indicated by recent acoustic absorption measurements on albumin solutions (Hushcha et al., 2002). The changes in polarizability entailed by conformational exchange might be large enough to lead to an optical coupling, albeit non-resonantly, of overdamped lowfrequency vibrations to microwave radiation. Thus, microwave fields are expected to influence structure and dynamics of proteins, if at all, only far from any resonance.

However, experimental investigations probing the response of protein structure and dynamics to microwave fields in well characterized in vitro model systems under controlled temperature conditions are still scarce and poorly understood: the unfolding and refolding of $\beta$-lactoglobulin in urea solution measured using optical rotation dispersion (ORD) has been observed to be accelerated by microwave irradiation at $v=2.45 \mathrm{GHz}$ (Bohr \& Bohr, 2000). Small-angle X-ray scattering from solutions of bovine serum albumine (BSA) was reported to show marked differences between samples that had been heated by microwaves at $v=2.45 \mathrm{GHz}$ and those heated in a water bath to the same temperature (Lerina et al., 1997), which was related to denaturation that could be obtained only by microwave heating. Recent measurements of light scattering from BSA solutions exposed to microwaves with $v=1.0 \mathrm{GHz}$ show, in accordance with the results by Lerina and coworkers, enhanced aggregation for microwaveexposed samples (de Pomerai et al., 2003). In a study of thermophilic $S$-adenosylhomocysteine hydrolase, the apparently non-thermal inactivation of the enzyme arising from irradiation at $10.4 \mathrm{GHz}$ was related to conformational changes revealed by fluorescence emission and ORD (Porcelli et al., 1997). A similar reduction of enzymatic activity and loss of helicity was observed for thermophilic alcohol dehydrogenase upon microwave irradiation at $10.4 \mathrm{GHz}$ using circular dichroism (La Cara et al., 1999). On the other hand, a study on myoglobin combining absorption spectroscopy at visible wavelengths, ORD and measurements of the tryptophan fluorescence lifetime showed no measurable differences before and after microwave irradiation at $1.95 \mathrm{GHz}$ (Bismuto et al., 2003).

In contrast to ORD and absorption spectroscopy at visible wavelengths, which probe gross structural features of the protein molecules, X-ray diffraction (XRD) from single protein crystals provides detailed information not only on the average atomic positions in a protein, but also on the meansquare displacements ( $B$ factors) of individual atoms. Enhanced mean-square displacements of protein atoms induced by resonant absorption of microwaves by intramolecular vibrational modes should thus be sensitively detectable as decreased Debye-Waller factors in the time-averaged X-ray diffraction images. This contribution presents detailed XRD measurements of atomic $B$ factors in tetragonal single crystals of hen egg-white lysozyme obtained under controlled continuous-wave microwave irradiation at $v=8 \mathrm{GHz}$. Our results show that high microwave power levels lead to increased, but largely recoverable, lattice defects arising from the evaporation of the crystal water. At power levels low enough that good-quality X-ray diffraction data can be recorded, the average $B$ factor is found to increase with microwave power, consistent with an increase in temperature. However, the $B$ factors of the atoms in a few highly localized sites in the protein show reproducible changes upon microwave irradiation that are not explained by heating.

\section{Materials and methods}

\subsection{Crystal growth and sample preparation}

Tetragonal $\left(\mathrm{P}_{3} \mathrm{2}_{1} 2\right)$ lysozyme crystals were grown following a standard procedure ${ }^{\mathbf{1}}$ and mounted in glass capillaries. Small reservoirs of $\mathrm{NaCl}$ solution at each end of the capillary prevented the crystals from drying. Two different types of samples in glass capillaries were prepared: crystals which were in direct contact with a small amount of mother liquor and crystals which had no direct contact with mother liquor, but were in equilibrium with the liquid reservoirs at the ends of the capillaries. The capillaries were sealed with wax and mounted on a goniometer.

\subsection{X-ray diffraction and data analysis}

$\mathrm{X}$-ray diffraction was carried out using a rotating-anode source (Schneider, Darmstadt, Germany) operated at $40 \mathrm{kV}$ and $100 \mathrm{~mA}$ at a wavelength of $1.54 \AA$. Diffractograms were recorded using a MAR 345 image plate (MAR Research, Norderstedt, Germany). Using a sample-to-detector distance of $120 \mathrm{~mm}$ and a plate diameter of $240 \mathrm{~mm}$ allows the recording of crystal reflections to a resolution of $2.0 \AA$. For the off-line experiments (see below), we recorded ten diffraction images during each of the $\Delta \varphi=1.5^{\circ}$ rotations about the goniometer axis perpendicular to the incoming X-ray beam. For the on-line experiments (see below) and the measurements at elevated temperature, complete data sets of 60 diffraction images $\left(\Delta \varphi=1^{\circ}\right)$ were recorded. Unit-cell parameters, Wilson $B$ factors, mosaicity and beam divergence were

\footnotetext{
${ }^{1}$ Lyophilized hen egg-white (HEW) lysozyme (Fluka) was crystallized at $291 \mathrm{~K}$ by the hanging-drop method. The drops were a mixture of $2 \mu$ lysozyme solution $\left(75 \mathrm{mg} \mathrm{ml}^{-1}\right)$ and $6 \mu \mathrm{l} \mathrm{NaCl}$ solution $[6 \%(w / v)]$. Both solutions were prepared in $0.1 M$ sodium acetate buffer $(\mathrm{pH} 4.8)$. The crystals reached a size of between $0.1 \mathrm{~mm}$ and $0.8 \mathrm{~mm}$ within two weeks.
} 
Table 1

Data-collection and refinement statistics for the data obtained from crystal 1 under microwave irradiation at varying power.

Values in parentheses refer to the highest resolution shell. The quantity $\sigma_{\mathrm{M}}$ is used as defined in $X D S$ (December 2002).

\begin{tabular}{|c|c|c|c|c|c|}
\hline Microwave power (W) & 0.0 & 1.0 & 1.0 & 3.0 & 3.0 \\
\hline Before/during/after & Before & During & After & During & After \\
\hline \multicolumn{6}{|l|}{ Data collection } \\
\hline$\sigma_{\mathrm{M}}\left({ }^{\circ}\right)$ & 0.120 & 0.183 & 0.132 & 0.144 & 0.177 \\
\hline Wilson $B$ factor $\left(\AA^{2}\right)$ & 25.87 & 25.92 & 26.30 & 28.01 & 27.92 \\
\hline \multicolumn{6}{|l|}{ Unit-cell parameters } \\
\hline No. of collected reflections & 37009 & 36466 & 36852 & 35604 & 36694 \\
\hline No. of unique reflections & 7402 & 7355 & 7378 & 7180 & 7304 \\
\hline Completeness (\%) & $85.8(80.0)$ & $85.3(79.7)$ & $85.5(80.0)$ & $84.0(79.6)$ & $84.4(76.6)$ \\
\hline$R_{\text {meas }}$ & $0.073(0.088)$ & $0.079(0.097)$ & $0.077(0.098)$ & $0.087(0.114)$ & $0.083(0.119)$ \\
\hline \multicolumn{6}{|l|}{ Refinement } \\
\hline Resolution $(\AA)$ & 2.00 & 2.00 & 2.00 & 2.00 & 2.00 \\
\hline No. of reflections used & 7046 & 7000 & 7023 & 6850 & 6958 \\
\hline \multicolumn{6}{|l|}{ Average $B$ factors } \\
\hline All non-H atoms $\left(\AA^{2}\right)$ & 22.79 & 22.64 & 22.97 & 25.03 & 24.83 \\
\hline Protein atoms $\left(\AA^{2}\right)$ & 20.50 & 20.33 & 20.68 & 22.84 & 22.57 \\
\hline Water $\mathrm{O}$ atoms $\left(\AA^{2}\right)$ & 38.85 & 38.83 & 39.11 & 40.43 & 40.72 \\
\hline $\mathrm{Na}^{+}\left(\AA^{2}\right)$ & 26.80 & 21.95 & 25.19 & 19.80 & 24.30 \\
\hline $\mathrm{Cl}^{-}\left(\AA^{2}\right)$ & 26.85 & 25.92 & 27.23 & 25.64 & 29.05 \\
\hline
\end{tabular}

Table 2

Data-collection and refinement statistics for the data obtained from crystal 2 under microwave irradiation at varying power.

Values in parentheses refer to the highest resolution shell.

\begin{tabular}{|c|c|c|c|c|c|c|}
\hline Microwave power (W) & 0.0 & 0.5 & 1.0 & 1.0 & 2.0 & 2.0 \\
\hline Before/during/after & Before & During & During & After & During & After \\
\hline \multicolumn{7}{|l|}{ Data collection } \\
\hline Resolution (Å) & $\begin{array}{l}50.00-2.00 \\
\quad(2.12-2.00)\end{array}$ & $\begin{array}{l}50.00-2.00 \\
\quad(2.12-2.00)\end{array}$ & $\begin{array}{l}50.00-2.00 \\
\quad(2.12-2.00)\end{array}$ & $\begin{array}{l}50.00-2.00 \\
\quad(2.12-2.00)\end{array}$ & $\begin{array}{l}50.00-2.00 \\
\quad(2.12-2.00)\end{array}$ & $\begin{array}{l}50.00-2.00 \\
\quad(2.12-2.00)\end{array}$ \\
\hline$I / \sigma(I)$ & $20.0(10.9)$ & $19.3(10.7)$ & $19.2(10.5)$ & $18.8(10.0)$ & $14.3(8.4)$ & $17.8(8.8)$ \\
\hline$\sigma_{M}\left({ }^{\circ}\right)$ & 0.087 & 0.089 & 0.088 & 0.088 & 0.099 & 0.089 \\
\hline Wilson $B$ factor $\left(\AA^{2}\right)$ & 26.42 & 26.65 & 26.87 & 27.29 & 27.90 & 28.14 \\
\hline \multicolumn{7}{|l|}{ Unit-cell parameters } \\
\hline$a(\AA)$ & 79.18 & 79.18 & 79.15 & 79.17 & 79.19 & 79.16 \\
\hline$c(\AA)$ & 37.98 & 37.99 & 37.99 & 37.99 & 38.03 & 38.00 \\
\hline No. of collected reflections & 37170 & 37129 & 37169 & 37209 & 36868 & 37280 \\
\hline No. of unique reflections & 8084 & 8080 & 8080 & 8084 & 8046 & 8088 \\
\hline Completeness (\%) & $93.7(80.7)$ & $93.7(80.5)$ & $93.8(80.9)$ & $93.8(81.0)$ & $93.6(81.4)$ & $93.0(76.1)$ \\
\hline$R_{\text {meas }}$ & $0.063(0.130)$ & $0.066(0.132)$ & $0.066(0.136)$ & $0.066(0.144)$ & $0.080(0.169)$ & $0.068(0.162)$ \\
\hline \multicolumn{7}{|l|}{ Refinement } \\
\hline Resolution (§) & 2.00 & 2.00 & 2.00 & 2.00 & 2.00 & 2.00 \\
\hline No. of reflections used & 7703 & 7699 & 7699 & 7703 & 7668 & 7700 \\
\hline$R$ & $0.128(0.130)$ & $0.127(0.134)$ & $0.129(0.133)$ & $0.129(0.136)$ & $0.131(0.141)$ & $0.131(0.139)$ \\
\hline$R_{\text {free }}$ & $0.194(0.243)$ & $0.191(0.248)$ & $0.193(0.241)$ & $0.194(0.264)$ & $0.189(0.198)$ & $0.171(0.251)$ \\
\hline \multicolumn{7}{|l|}{ R.m.s.d. from ideality } \\
\hline Bonds $(\AA)$ & 0.01 & 0.01 & 0.01 & 0.01 & 0.01 & 0.01 \\
\hline Angles $\left(^{\circ}\right)$ & 1.305 & 1.293 & 1.294 & 1.311 & 1.292 & 1.303 \\
\hline \multicolumn{7}{|l|}{ Average $B$ factors } \\
\hline All non-H atoms $\left(\AA^{2}\right)$ & 25.01 & 24.83 & 25.08 & 25.76 & 25.93 & 26.75 \\
\hline Protein atoms $\left(\AA^{2}\right)$ & 22.76 & 22.59 & 22.87 & 23.53 & 23.66 & 24.45 \\
\hline Water $\mathrm{O}$ atoms $\left(\AA^{2}\right)$ & 40.82 & 40.60 & 40.67 & 41.45 & 41.89 & 42.9 \\
\hline $\mathrm{Na}^{+}\left(\AA^{2}\right)$ & 30.61 & 27.38 & 23.94 & 28.72 & 22.35 & 29.94 \\
\hline $\mathrm{Cl}^{-}\left(\AA^{2}\right)$ & 30.38 & 29.59 & 28.90 & 30.66 & 29.30 & 30.97 \\
\hline
\end{tabular}

determined using the program $X D S$ (Kabsch, 2001a,b). The Protein Data Bank structure of HEW lysozyme (PDB code
1931; resolution 1.33 Å; Vaney et al., 1996) was refined against the data recorded during microwave irradiation using the 
Table 3

Data-collection and refinement statistics for the data obtained from crystal 3 at varying temperatures.

Values in parentheses refer to the highest resolution shell.

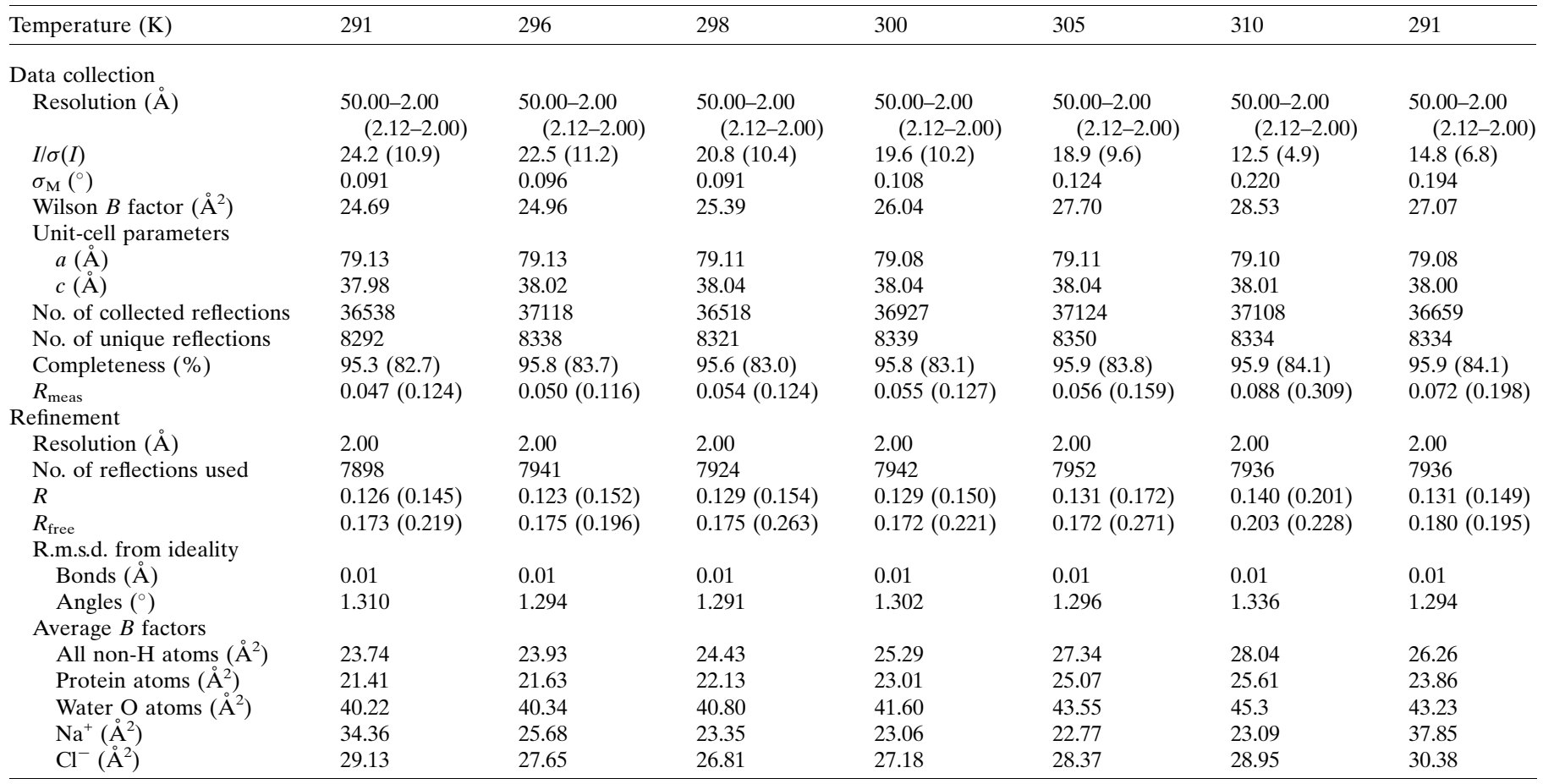

program REFMAC5 from the CCP4 suite v.4.2 (Collaborative Computational Project, Number 4, 1994).

This analysis yields the atomic coordinates and $B$ factors of the 1001 non-H atoms in lysozyme as well as those of two ions $\left(\mathrm{Na}^{+}\right.$and $\left.\mathrm{Cl}^{-}\right)$and 142 strongly bound water molecules. Details of the crystal structure and the refinement are given in Tables 1, 2 and 3.

\subsection{Microwave irradiation}

On-line XRD during microwave irradiation was carried out using a modified slab-line waveguide (Weissenborn et al., 2004). A single-frequency continuous-wave signal at $v=8 \mathrm{GHz}$ was generated using a swept-signal generator (Micro-Tel Corporation SG-811B); after amplification by a travellingwave tube amplifier (Varian 6991K3), the signal was coupled into the waveguide. The transmitted power was attenuated and measured with a power meter (Hewlett-Packard 432A with power sensor $8478 \mathrm{~B})$. Crystals were placed at the position of maximal field strength located in the $1.25 \mathrm{~mm}$ wide gap between the central and outer conductor. A time-domain finite-difference calculation of the electric field inside the empty waveguide for $P=1 \mathrm{~W}$ input power at $v=2 \mathrm{GHz}$ yielded values of $|E|=9.5 \mathrm{kV} \mathrm{m}^{-1}$ and $S=0.12 \mathrm{~W} \mathrm{~mm}^{-2}$ for the magnitude and the intensity of the electric field at the crystal location. Upon inserting an empty capillary into the waveguide the transmitted power was found to remain constant, showing that the electric field inside the waveguide is not significantly distorted by the dielectric discontinuity introduced by the capillary and the crystal. The salt-solution reservoirs in each end of the capillary were located outside the slab-line and were thus not exposed to the microwave field.

Irradiation experiments were then carried out following two different protocols: in order to determine a threshold microwave power level that would lead to thermally induced crystal damage, crystals were exposed to increasing power levels at constant irradiation time $\tau=10 \mathrm{~min}$; XRD was performed offline before and after the irradiation. For each power level we used a new lysozyme crystal.

In the second set of experiments, complete XRD data sets were recorded on-line during continuous irradiation of crystals at power levels below the damage threshold. The irradiation lasted for $\tau=4 \mathrm{~h}$ at $v=8 \mathrm{GHz}$ and incident power levels $P \leq 3 \mathrm{~W}$, well below the power levels leading to dehydration effects. Crystal 1 was consecutively exposed to $P=1$ and $3 \mathrm{~W}$ and crystal 2 was irradiated with $P=0.5,1$ and $2 \mathrm{~W}$ in the given order. As a control, we recorded complete data sets before and after the microwave treatment for both crystals.

\subsection{Temperature-dependent XRD}

In order to study the effect of elevated temperature on the structure and atomic mean-square displacements of HEW lysozyme, complete XRD data sets were recorded in the absence of microwave irradiation at temperatures between 291 and $310 \mathrm{~K}$. The temperature of the crystal and the liquor reservoir was controlled by directing the gas stream from a nitrogen cooler (Oxford 600 Series Cryostream) onto the capillary containing the protein crystal. 


\subsection{Accuracy of the measurement and refinement procedure}

In order to estimate the statistical error in the $B$-factor values obtained from the measurement and refinement procedure, we recorded two complete data sets from the same crystal at $291 \mathrm{~K}$ against which the PDB structure 1931 was refined. The distribution of the $B$-factor differences between the two data sets was a Gaussian centred at $\langle\Delta B\rangle=0.18 \pm$ $0.01 \AA^{2}$ with a standard deviation $\sigma_{\Delta B}=0.8 \AA^{2}$. We thus use this standard deviation as the lower limit for the detection of atomic $B$-factor differences between data sets obtained from the same crystal.

The shift of the centre of the Gaussian is consistent with the increase of the Wilson $B$-factor value from 22.80 to $22.98 \AA^{2}$. We ascribe this increase to radiation damage incurred by the crystal. Since the integration time $\tau=4 \mathrm{~h}$ and the intensity of the X-ray beam during all the experiments discussed in $\$ \$ 3.2$ and 3.3 were identical and assuming that the radiation damage increases linearly with the absorbed X-ray dose, we use the value $\langle\Delta B\rangle=0.18 \pm 0.01 \AA^{2}$ as an estimate for the increase in the radiation damage between two consecutive data sets.

\section{Results}

\subsection{Thermal damage threshold}

Thermal damage to the protein crystal induced by microwave power is most clearly revealed by the estimated standard deviation (e.s.d.) of the beam divergence $\sigma_{\mathrm{D}}$ and the reflecting range e.s.d. $\sigma_{\mathrm{M}}$ as defined in $X D S$ (Kabsch, 2001 $a, b$; see Fig. 1). The former quantity represents the angular spread of the Bragg spots arising from instrumental divergence of the X-ray beam and from the size and the mosaicity of the crystal, while the latter represents the mosaicity alone. Determining $\sigma_{\mathrm{D}}$ and $\sigma_{\mathrm{M}}$ with $X D S$ yielded reproducible results across the ten diffraction images of a data set since the number of unique reflections in each diffraction image was about 600 .

At low power $P<4 \mathrm{~W}$ and an irradiation time $\tau=10 \mathrm{~min}$ the values of $\sigma_{\mathrm{D}}$ and $\sigma_{\mathrm{M}}$ are constant at about $0.1^{\circ}$. For higher $P$, the values of $\sigma_{\mathrm{D}}$ and $\sigma_{\mathrm{M}}$ increase sharply to about 0.17 and $0.6^{\circ}$, respectively, when the crystals were immersed in a mother-liquor drop. These crystals cracked, turned opaque and did not recover after microwave exposure. Finally, at microwave power levels $P>9 \mathrm{~W}$ the diffraction quality was so poor that quantities such as $\sigma_{\mathrm{D}}$ or $\sigma_{\mathrm{M}}$ could no longer be determined.

In contrast, crystals which were not in direct contact with the mother liquor ('dry' crystals) showed constant $\sigma_{\mathrm{D}}$ and $\sigma_{\mathrm{M}}$ up to a power $P=7 \mathrm{~W}$. Higher microwave power levels also deteriorated the diffraction properties of these crystals, but only temporarily. For $P=15$ and $20 \mathrm{~W}$ the crystals showed a few large-scale cracks. Interestingly, soon after irradiation the diffraction patterns of these crystals recovered almost completely (see Fig. 2). We waited at least $45 \mathrm{~min}$ to let the crystals recover before recording another data set from the crystals which had been irradiated with $P=10,15$ and $20 \mathrm{~W}$. The values of $\sigma_{\mathrm{D}}$ for these data sets are only slightly increased, to about $0.12^{\circ}$ at most (see Fig. $1 a$ ).
The observed recovery of the diffraction pattern after the microwave treatment probably arises from reversible, continuous dehydration of the crystal: irradiation leads to crystal shrinkage as weakly bound water residing in the pores of the crystal structure evaporates. However, shrinkage of the crystal lattice is likely to be compensated by a large amount of elastic energy, which will provide a substantial driving force for rehydration in the absence of irradiation. We attribute this behaviour to thermal effects, since we also observed it after heating a crystal without mother-liquor contact in an oil bath at a temperature of $320 \mathrm{~K}$ for $10 \mathrm{~min}$. The recovery of the crystal order after dehydration is also in line with the recent observation of structural reversibility upon hydration changes by means of ambient humidity control (Kiefersauer et al., 2000; Dobrianov et al., 2001).

Unlike $\sigma_{\mathrm{D}}$ and $\sigma_{\mathrm{M}}$, the changes in the Wilson $B$ factor (which is the $B$ factor averaged over all atoms) and the two unit-cell parameters $a$ and $c$ (see Fig. $3 a$ ) were only minor, even at power levels high enough to cause severe damage to the crystal. While the Wilson $B$ factor of 'wet' crystals shows a slight non-monotonic behaviour with microwave power

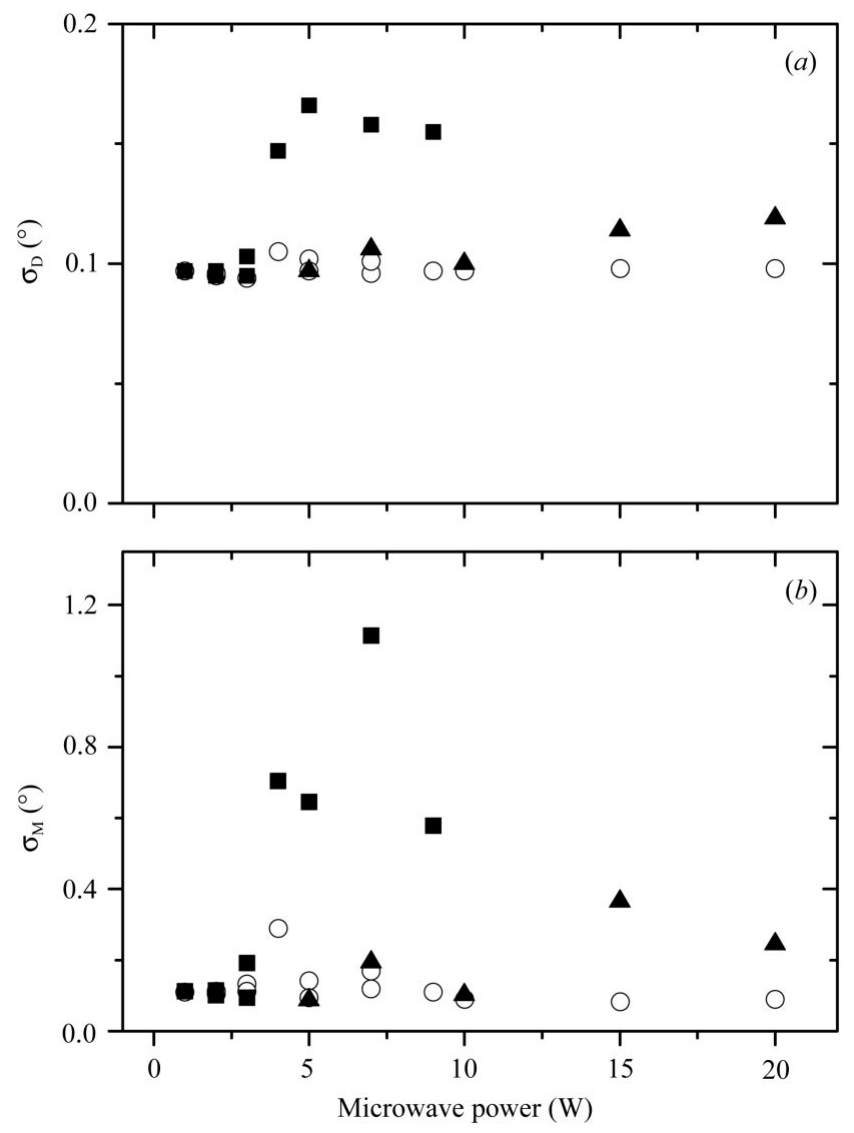

Figure 1

(a) Beam-divergence estimated standard deviation $\sigma_{\mathrm{D}}$ and (b) reflectingrange estimated standard deviation $\sigma_{\mathrm{M}}$ (mosaicity) of lysozyme crystals irradiated with microwaves with a frequency $v=8 \mathrm{GHz}$ for periods $\tau=10 \mathrm{~min}$ as a function of incident microwave power $P$. Open circles, before irradiation; squares, crystals immersed in a drop of mother liquor; triangles, crystals without mother-liquor contact. The data points at $P=10,15$ and $20 \mathrm{~W}$ for the crystals without mother-liquor contact are values determined after the crystals have recovered from dehydration. 


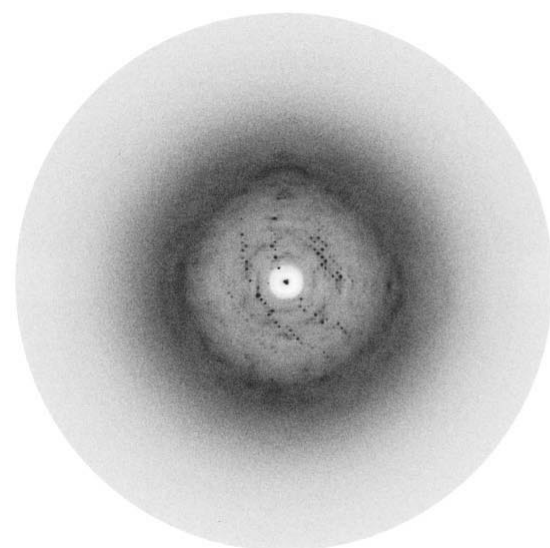

(a)

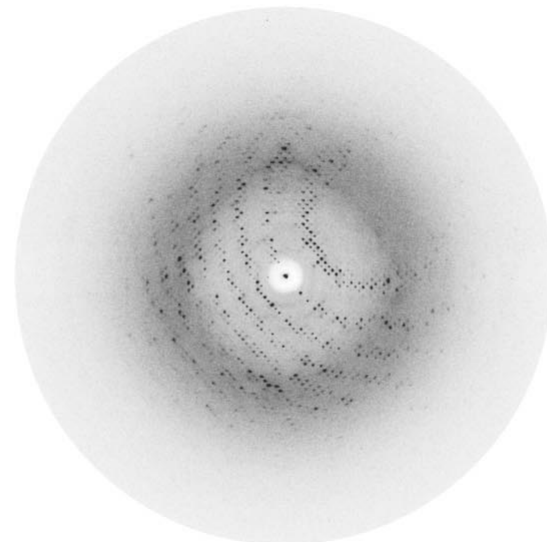

(b)

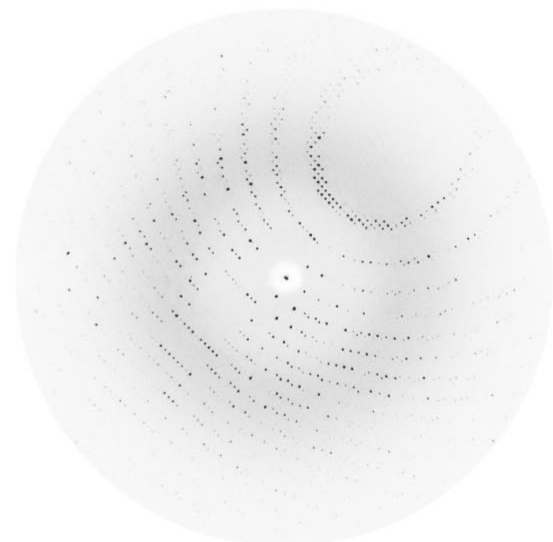

(c)

Figure 2

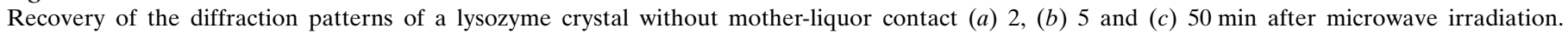

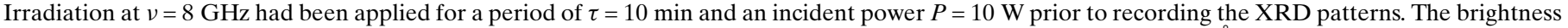

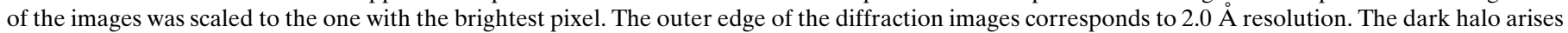

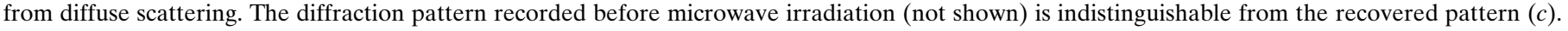

(possibly owing to uncontrolled temperature increases resulting from variations in the volume of surrounding liquid), it hardly changes for 'dry' crystals (see Fig. $3 b$ ).
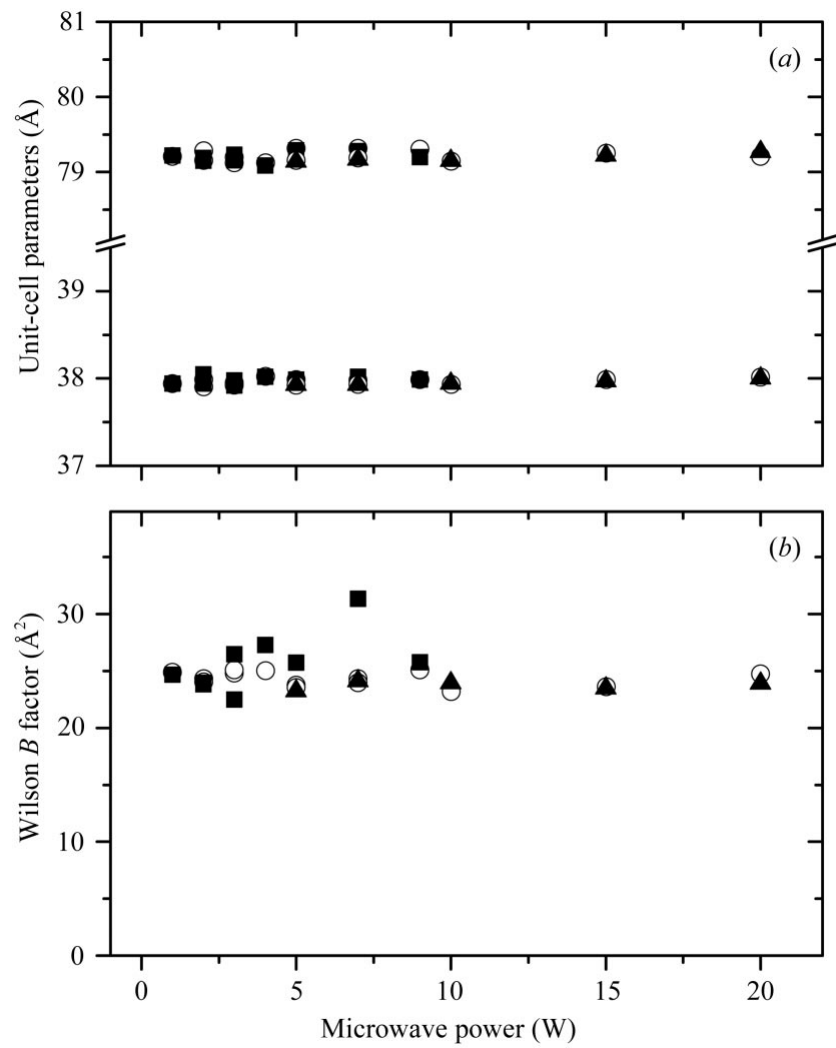

Figure 3

(a) Unit-cell parameters $a=b$ (upper symbols) and $c$ (lower symbols) and (b) Wilson $B$ factors of lysozyme crystals irradiated with microwaves with frequency $v=8 \mathrm{GHz}$ for periods $\tau=10 \mathrm{~min}$ as a function of incident power $P$. Open circles, before irradiation; squares, crystals immersed in a drop of mother liquor; triangles, crystals without mother-liquor contact. The data points at $P=10,15$ and $20 \mathrm{~W}$ for the crystals without motherliquor contact are values determined after the crystals had recovered from dehydration.
These findings indicate that microwave radiation is absorbed mainly by the crystal water and the free bulk water around the crystal. If the protein crystal is heated too much or too fast by the absorbing water $(P>4 \mathrm{~W})$, it cracks owing to thermal stress and thus shows significantly higher mosaicity, whereas its fragments still show good crystal order, as can be seen from the nearly constant Wilson $B$ factor and unit-cell parameters. On the other hand, if the crystal is not in contact with surrounding water the crystal is dehydrated during microwave irradiation owing to crystal water evaporation. This leads to shrinkage and loss of crystal order. In this state, a crystal can survive $10 \mathrm{~min}$ at a microwave power of up to $20 \mathrm{~W}$ without losing the ability to recover by rehydration afterwards.

\subsection{On-line microwave irradiation with non-destructive power levels}

3.2.1. Global features. A global picture of the effect of microwave irradiation on the mean-square displacements can be obtained by plotting $B(P)$, the $B$ factors during the irradiation, versus those measured before the irradiation, $B_{0}$, for each atom in the protein (see Fig. $4 a$ ). A purely thermal effect of microwave irradiation on the dynamics of the protein arising from overall heating of the crystal to some temperature $T$ above room temperature $T_{0}$ should then result in an enhancement of the $B$ factor of each atom by a factor $T / T_{0}$, provided the force fields governing the atomic mean-square displacements are harmonic. The correlation plot should then follow a linear behaviour $B(P)=\alpha+\beta B_{0}$, with an intercept $\alpha \simeq 0$ and a slope $\beta=T / T_{0}$. However, the experimental correlation plots look different: for all the microwave power levels investigated, the distribution of protein atom $B$ factors during irradiation is wider than that without irradiation. This widening is not only a consequence of the global enhancement of the $B$ factors and experimental uncertainties, but also of the presence of a number of atoms whose $B$ factors are increased 
beyond or decreased below the average during irradiation. The slope $\beta$ of the correlation plot between $B_{0}$ and $B(P)$ is very close to 1 for all microwave power levels investigated and does not measurably depend on microwave power.

The intercept $\alpha$, on the other hand, is found to increase monotonically with microwave power and reaches about $2.6 \AA^{2}$ at $P=3 \mathrm{~W}$ for crystal 1 and $1.4 \AA^{2}$ at $P=2 \mathrm{~W}$ for crystal 2 (see Fig. 5). $B$ factors measured after the irradiation do not entirely recover to their values measured before irradiation, as shown by the intercepts $\alpha=2.3 \AA^{2}$ for crystal 1 and $\alpha=1.1 \AA^{2}$ for crystal 2 obtained from the correlation analysis of the $B$ factors measured after microwave irradiation and those measured before the first microwave irradiation (see Fig. 4b). This indicates that the microwave-induced changes in the mean-square displacements are only partially reversible.

This incomplete reversibility cannot be explained by radiation damage alone. The increase in $\alpha$ owing to radiation damage should be at most $0.18 \AA^{2}$ between each pair of consecutive measurements (see $\$ 2.5$ ). For crystal 1 this gives a contribution of $0.72 \AA^{2}$ after the microwave irradiation with $P=3 \mathrm{~W}$ and for crystal 2 , which was irradiated one time more than crystal 1 , this value is $0.90 \AA^{2}$ after irradiation with $P=2 \mathrm{~W}$. This shows that the value of the intercept $\alpha$ is mainly determined by the microwave power and to a lesser extent by the radiation damage from successive measurements.

3.2.2. Detailed features. While the correlation plots shown above provide information on the dependence of the average $B$ factor on microwave power, more detailed information on the effect of microwave irradiation is revealed by calculating the difference $\Delta B(P)=B(P)-B_{0}$ between the $B$ factors measured during irradiation and those measured before irradiation for each non-H atom individually. Plotted as a function of atom number, $\Delta B(P)$ shows an irregular pattern which is a fingerprint of the effect of microwave irradiation on the atomic mean-square displacements.

As shown in Fig. 6, microwave irradiation leads to large changes in the $B$ factors only at localized sites within the protein. It is interesting to note that microwave irradiation may also lead to a local decrease in $B$ factors. For $P=2.0 \mathrm{~W}$, we find that about $58 \%$ of all non- $\mathrm{H}$ protein atoms have $\Delta B$ values larger than the experimental noise level of $0.4 \AA^{2}$, compared with $20 \%$ of all protein atoms whose $\Delta B$ values are smaller than $-0.4 \AA^{2}$.

Also shown in Fig. 6 are the differences between the protein atom $B$ factors at elevated temperature $T=298 \mathrm{~K}$ and those measured at $291 \mathrm{~K}$. This data set shows a similar irregular pattern with a small number of large values and, similar to the microwave data, also strongly decreased $B$ factors at a few sites within the protein. In general, the $B$-factor differences observed at higher temperature agree closely with those observed during microwave irradiation.

In order to understand the origin of the large negative values of $\Delta B$ that we observe at a few sites, we consider the two regions where the decrease in the $B$ factors upon microwave irradiation is most pronounced: (i) in the region between residues 66 and 77 (see Fig. 7) the atoms with the largest $\Delta B$ are the backbone $\mathrm{O}$ in Gly71, Ser72 OG and the backbone $\mathrm{O}$ in Arg73. Together with Ser60 O, Cys64 O (these two atoms show negligible $\Delta B$ values) and two water molecules, the latter two atoms are coordinated to an $\mathrm{Na}^{+}$ion in a distorted octahedral configuration (Vaney et al., 1996).

The backbone $\mathrm{O}$ atom of $\mathrm{Arg} 73$ shows a $B$ factor reduced by $8.7 \AA^{2}$ for $P=2 \mathrm{~W}$. Large decreases in the $B$ factor at this site are already seen at the lowest microwave power. When the $B$ factor of the backbone $\mathrm{O}$ of Arg73 is measured after microwave irradiation, it is found to partially relax to a value about $2.5 \AA^{2}$ smaller than the value before irradiation. This effect has been observed in two crystals independently. After the microwave treatment, the $B$ factors around Ser72 are found to recover only incompletely, to values about $4.0 \AA^{2}$ smaller than those before irradiation. The $\mathrm{Na}^{+}$ion shows a similar decrease of its $B$ factor with increasing microwave power to its two ligands Ser72 OG and the backbone $\mathrm{O}$ in Arg73 (see Tables 1 and 2).

In the second region, spanning residues $11-15$ (see Fig. 7), the atoms with the largest negative $\Delta B(P)$ are the terminal side-chain $\mathrm{N}$ atoms in Arg14, a residue located on the surface of the protein. Here, the $B$ factors are reduced by as much as 6.4 and $7.8 \AA^{2}$ at $2 \mathrm{~W}$ microwave power. Unlike Ser72 and $\operatorname{Arg} 73$, where the decrease of $B$ is already detectable at the lowest microwave power of $0.5 \mathrm{~W}$, the reduction of $B$ at Arg14 only shows up at the highest power level of $2 \mathrm{~W}$. We also detected this decrease in the second crystal investigated; here, a reduction in the $B$ factor of Arg14 NH1 by $3.7 \AA^{2}$ could already be detected for $P=1.0 \mathrm{~W}$. In contrast to the $B$ factors near Ser72, those in Arg14 are found to relax back to their values before irradiation in the absence of microwaves.

\subsection{Temperature variation: comparison with microwave irradiation}

In order to answer the question whether the strongly reduced atomic mobility at Arg14 and Ser72 during microwave irradiation arises from a rise in temperature induced by microwave absorption by the crystal water, we have analyzed the $B$-factor patterns measured from a tetragonal lysozyme crystal at temperatures between 291 and $310 \mathrm{~K}$ in the absence of microwave irradiation. Details of the crystal structures and the refinements are given in Table 3.

A correlation analysis analogous to that presented above for the microwave data can be applied to the $B$ factors measured at elevated temperatures: between $T_{1}=296 \mathrm{~K}$ and $T_{2}=305 \mathrm{~K}$ the slope $\beta(T)$ of the correlation plot of $B(T)$ versus $B(291 \mathrm{~K})$ is expected to increase by only $3 \%$, which is at the limit of what we are able to resolve with the current data.

On the other hand, the intercept $\alpha(T)$ increases linearly with temperature, providing a sensitive thermometer by which the temperatures of the microwave-irradiated crystals can be estimated (see Fig. 5). By this method, we estimate that the highest non-destructive microwave power level $P=3 \mathrm{~W}$ leads to a temperature of about $304 \mathrm{~K}$.

After the measurements at elevated temperatures the intercept $\alpha(310 \mathrm{~K})=4.4 \AA^{2}$ recovers to a value of $\alpha(291 \mathrm{~K})=$ $1.77 \AA^{2}$. This value can largely be explained by the estimated 
increase by $1.1 \AA^{2}$ arising from radiation damage (see $\$ 2.5$ ) after the seven successive measurements.

Focusing on the region around Ser72, one of the regions where the $B$ factors are reduced during microwave irradiation, the $\Delta B$ patterns are similar to those observed during microwave irradiation: at Gly71, Ser72 and $\operatorname{Arg} 73$ the $B$ factors are reduced by up to $17.3 \AA^{2}$ when the temperature is increased by $19 \mathrm{~K}$ above room temperature (see Fig. 8). For all these sites with strong response to temperature changes we observe a monotonic decrease of $B$ with temperature. This pattern bears a strong resemblance to the pattern observed during microwave irradiation, indicating that the microwave response of the $B$ factors at this site is thermal in origin.

In contrast, the $B$ factors at Arg14 increase weakly with temperature, the opposite of the behaviour observed under microwave irradiation, except for $\operatorname{Arg} 14 \mathrm{NH} 2$, for which the $B$ factor is reduced.

\section{Discussion}

The above analysis shows that irradiating tetragonal lysozyme crystals with intense microwave fields leads to significant

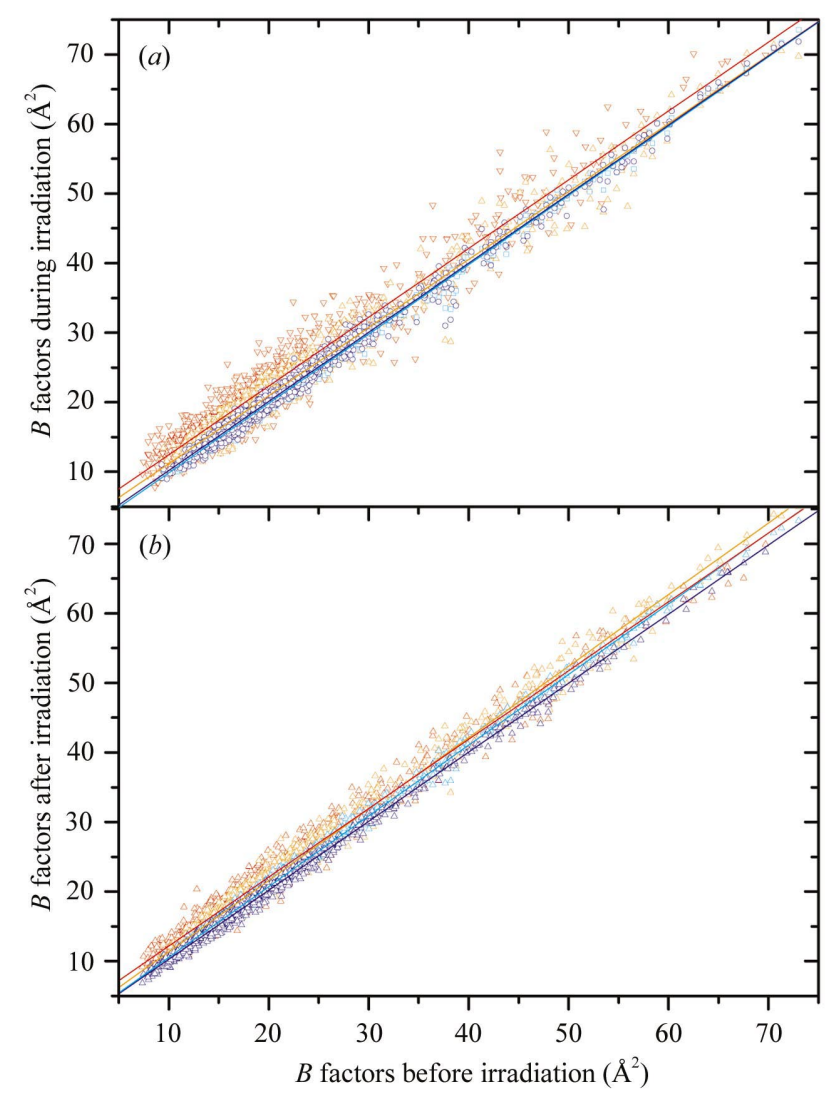

Figure 4

Correlation plots of the $B$ factors of 1001 protein atoms measured from two lysozyme crystals $(a)$ before $\left(B_{0}\right)$ and during $[B(P)]$ the microwave irradiation with $v=8 \mathrm{GHz}, P=0.5 \mathrm{~W}$ (cyan), $1 \mathrm{~W}$ (blue), $2 \mathrm{~W}$ (orange) and $3 \mathrm{~W}$ (red). (b) Correlation plot of the $B$ factors measured before $\left(B_{0}\right)$ and after $[B(P=0 \mathrm{~W})]$ the microwave irradiation. Symbols are as in $(a)$. The lines represent linear fits $B(P)=\alpha+\beta B_{0}$ for $(a)$ and $B(P=0 \mathrm{~W})=\alpha+$ $\beta B_{0}$ for $(b)$, with the intercept $\alpha$ characterizing the overall increase in the atomic mean-square displacements. largely reversible changes in the atomic mean-square displacements not only at the ends of the backbone but also in other residues located near the surface of the protein. On average, atomic $B$ factors increase with both microwave power and temperature, apart from some highly localized sites whose $B$ factors decrease upon irradiation or increasing temperature. Such behaviour has also been reported for orthorhombic lysozyme crystals (Joti et al., 2002) at low temperatures $160<T<200 \mathrm{~K}$. This anomalous behaviour was explained by large intramolecular coordinate shifts arising from a structural phase transition of ice at $150 \mathrm{~K}$, entailing a structural phase transition in the protein and a redistribution of conformational substates. At the temperatures at which we carried out our experiments, tetragonal lysozyme does not undergo a

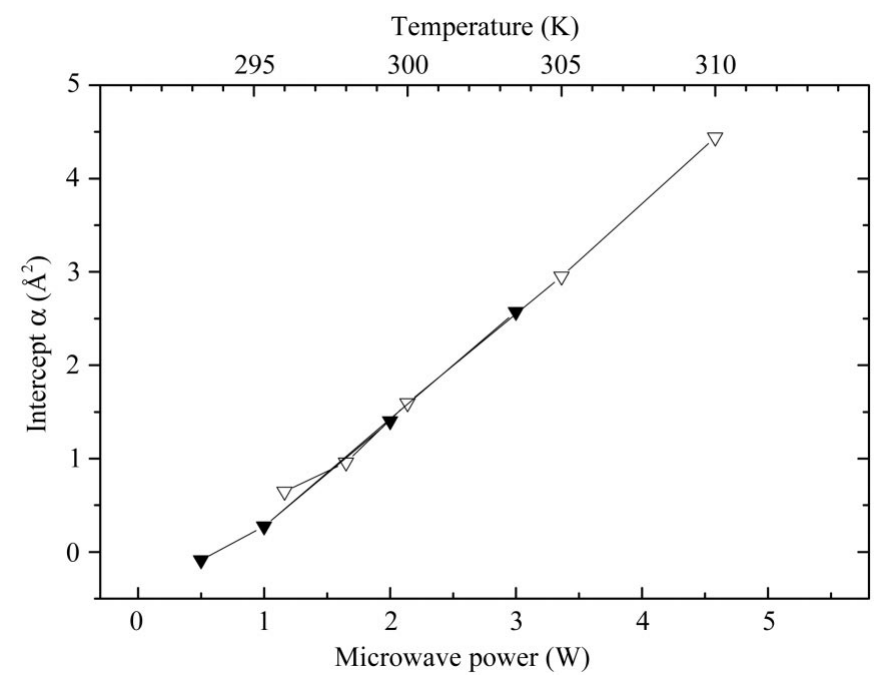

Figure 5

Calibration of the microwave-induced temperature increase by the intercept $\alpha$ from the linear-correlation analysis of $B(T)$ versus $B(291 \mathrm{~K})$ (open symbols). The full symbols are the irradiation-power dependent values of $\alpha$ obtained from the correlation analysis of the $B$ factors during microwave irradiation versus those before irradiation.

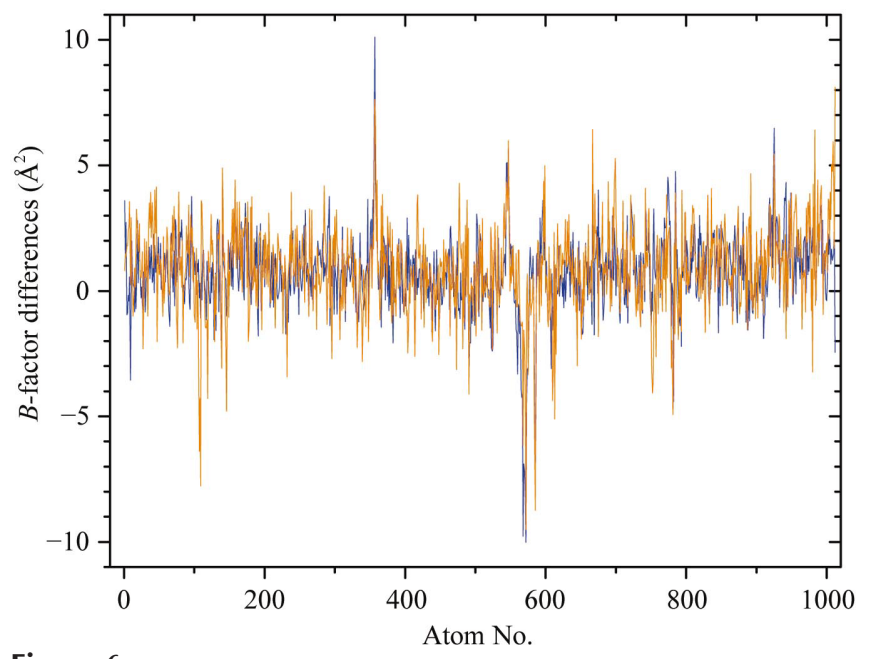

Figure 6

Difference between the $B$ factors of the non-H protein atoms in HEW lysozyme measured during microwave irradiation with an average power of $2.0 \mathrm{~W}$ and those before irradiation as a function of atom number (orange). The blue line is the difference between the $B$ factors measured at $T=298 \mathrm{~K}$ and those measured at $291 \mathrm{~K}$. 
structural phase transition, so the origin for the anomalous behaviour of some $B$ factors found here must be sought elsewhere.

While most of the $B$-factor changes during microwave irradiation can be explained by increased temperature, the large decrease in the $B$ factor of the terminal $\mathrm{N}$ atoms in Arg14 is absent in the data obtained at elevated temperature. It is tempting to interpret this highly local feature in the atomic mobility pattern as a fingerprint of a non-thermal response to the microwave field. We think, however, that a more likely explanation for the different response of Arg14 to microwave irradiation and to the elevated temperature is a subtle difference in the experimental conditions: in contrast to the measurements at elevated temperature (in the absence of microwave irradiation) where the entire capillary containing the protein crystal and the liquor reservoir were at roughly the

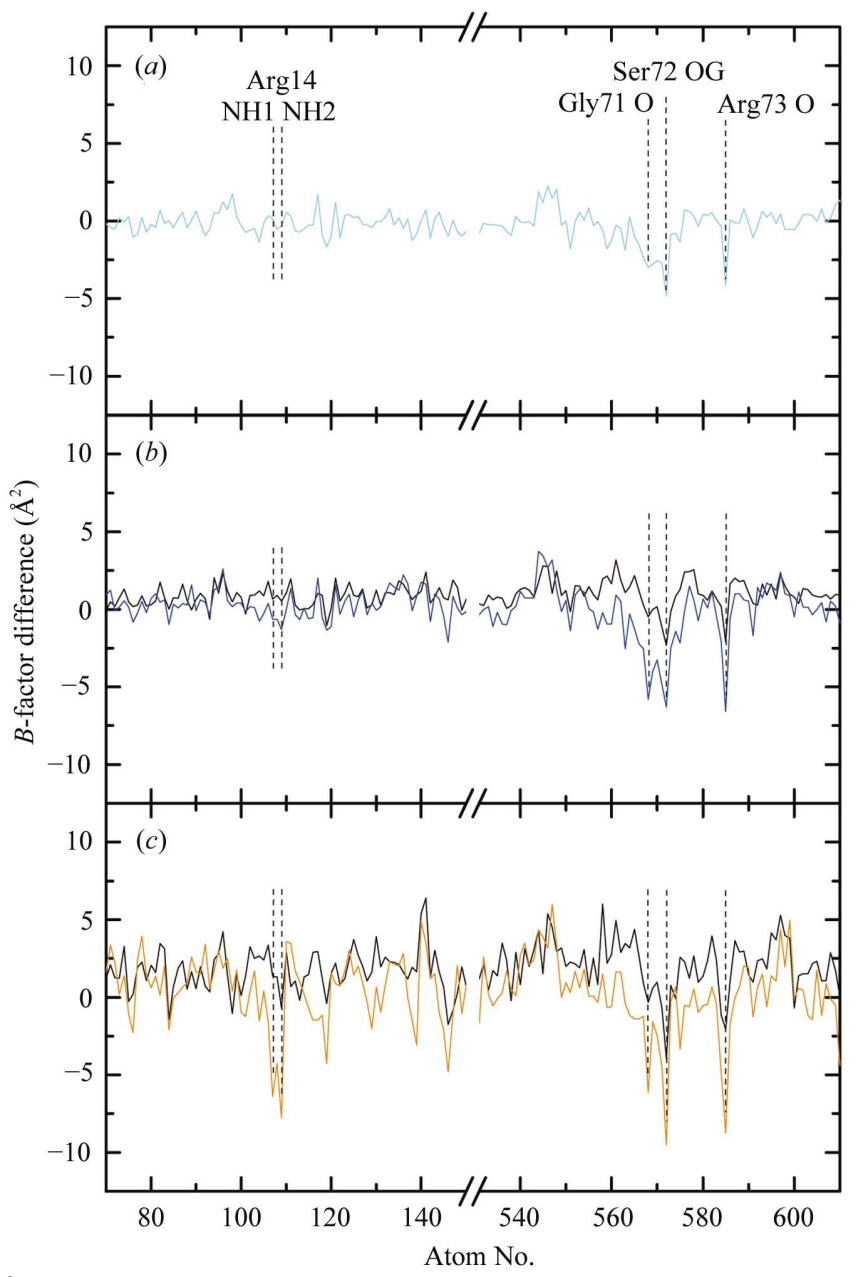

Figure 7

$B$-factor differences $\Delta B(P)$ of non-H protein atoms in residues 9-19 and 66-77 of HEW lysozyme as a function of the atom number measured during microwave irradiation with average power (a) $0.5 \mathrm{~W}$ (cyan), (b) $1.0 \mathrm{~W}$ (blue) and $(c) 2.0 \mathrm{~W}$ (orange). The black lines are the $B$-factor differences measured after each irradiation. The reference data are the $B$ factors measured before microwave irradiation. Lines denote the positions of Arg14 NH1 and NH2, Gly71 O, Ser72 OG and Arg73 O. The latter two are part of the distorted octahedral coordination complex of the $\mathrm{Na}^{+}$ion containing in addition Ser60 O, Cys64 O and two water molecules. At Arg14, the microwave-induced reduction of the $B$ factors is reversible, while at Ser72 and Arg73 the recovery is incomplete. same temperature, the experimental geometry in the irradiation experiments led to selective heating of the crystal, while the mother-liquor reservoirs located outside the waveguide remained at room temperature. This could have resulted in slight reversible dehydration of the crystal, driven by a gradient in water vapour pressure, predominantly affecting the atoms located at the periphery of the protein. Such a scenario would suggest that the large decrease in mobility of the terminal $\mathrm{N}$ in $\operatorname{Arg} 14$ is a consequence of a changed neighbourhood resulting in stronger force constants. This would imply significant changes in the average structure near Arg14 during microwave irradiation. However, within experimental error, we have not been able to identify such structural changes at or near Arg14.

As we show, the absorption of microwave power leads to temperatures which are, at the highest microwave irradiation levels used, no more than $304 \mathrm{~K}$. A rough estimate of the absorbed microwave energy, using the microwave absorption coefficient $0.192 \mathrm{~mm}^{-1}$ of hydrated lysozyme at $25 \mathrm{GHz}$ (Harvey \& Hoekstra, 1972), yields a rapid temperature increase of about $9 \mathrm{~K} \mathrm{~s}^{-1}$ for a microwave intensity of $0.12 \mathrm{~W} \mathrm{~mm}^{-2}$ (corresponding to a total input power $P=1 \mathrm{~W}$; Weissenborn et al., 2004) at $v=8 \mathrm{GHz}$. Such a heating rate would lead to thermal denaturation within a few seconds, contrary to the moderate temperature increase of $13 \mathrm{~K}$ observed in the experiment. Heat conduction via the partially wetted capillary walls and convection at the crystal surface are thus likely mechanisms that prevent the crystals from being excessively heated. However, a quantitative estimate of the effect of heat conduction and convection is difficult given the poorly known masses of the crystal and the surrounding water.

The sensitive dependence of the intercept $\alpha(T)$ of the correlation plots on temperature suggests that the main contribution to this quantity might come from acoustic phonons in the crystal. Owing to their long wavelength compared with the diameter of a lysozyme molecule, these provide a uniform contribution to the $B$ factors of all atoms in

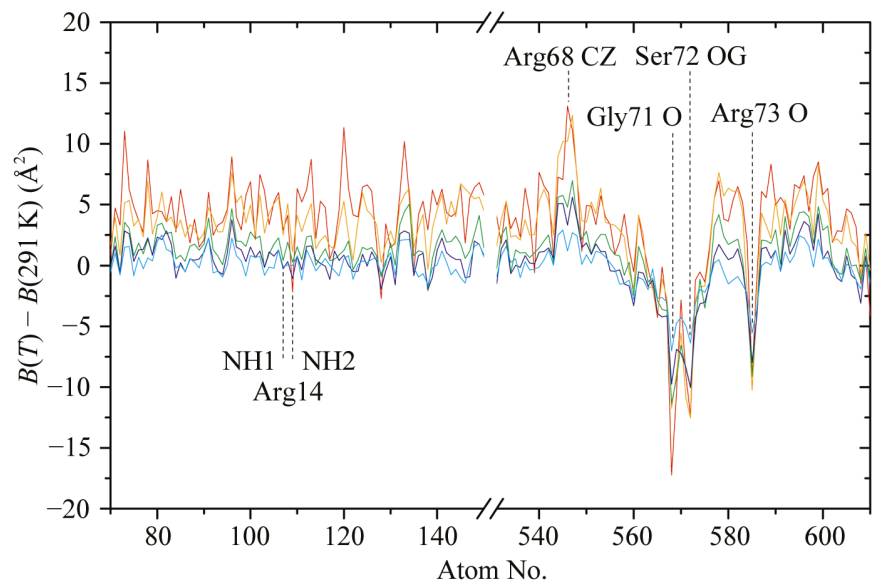

Figure 8

$B$-factor differences $\Delta B(T)$ of non-H protein atoms in residues 9-19 and 66-77 of HEW lysozyme as a function of atom number measured at temperatures of $296 \mathrm{~K}$ (cyan), $298 \mathrm{~K}$ (blue), $300 \mathrm{~K}$ (green), $305 \mathrm{~K}$ (orange) and $310 \mathrm{~K}$ (red). The reference data are the $B$ factors measured at room temperature $(291 \mathrm{~K})$. 
a unit cell. However, a rough estimate based on the Debye model for the density $g(\omega)$ of acoustic vibrational modes, using a value for the longitudinal sound speed $v_{\mathrm{s}}=2200 \mathrm{~m} \mathrm{~s}^{-1}$ of hydrated crystals obtained from Brillouin scattering ( $R$. Weissenborn, G. Maret and T. Gisler, unpublished results), yields values for the contribution of acoustic phonons to the $B$ factors between $0.23 \AA^{2}$ at $T=291 \mathrm{~K}$ and $0.25 \AA^{2}$ at $T=310 \mathrm{~K}$, which are much too small to account for the observed temperature dependence of the Wilson $B$ factors or the correlation intercepts $\alpha(T)$. Possible origins of this discrepancy could be the neglect of transverse acoustic modes, dispersion in the speed of sound or non-Debye behaviour related to anharmonic intermolecular potentials. Properly accounting for such effects should, however, correct the contribution of the unit-cell motion to the $B$ factors by no more than a factor of 2 . It is thus much more likely that the observed strong increase in the $B$ factors, the Wilson $B$ factor and the intercept $\alpha$ with microwave power and temperature arises from lattice defects originating in heating-induced slight rearrangements of the protein molecules in the crystal. These defects could also be irreversible, in particular if the temperature rise is not homogeneous throughout the crystal or if the crystal is not in equilibrium with the surrounding vapour of the reservoirs in both ends of the capillary, as discussed above.

\section{Conclusions}

In conclusion, we have used X-ray diffraction under simultaneous irradiation with microwaves to study changes in the atomic mobility and structure of the globular protein lysozyme. At power levels too small to induce significant lattice defects, we find that the atomic $B$ factors increase on average with increasing microwave power, while at some localized sites the $B$ factors are found to decrease with increasing microwave power. The similarity of these patterns with those measured at elevated temperature indicates that most of the changes in atomic mean-square displacements arise from heating of the crystal owing to microwave absorption by unbound water. A significant difference between microwave and temperature effects is found at the side chain of the peripheral residue Arg14, where the $B$ factors decrease with increasing microwave power but not with increasing temperature. While slight dehydration of the crystal owing to the microwave treatment could possibly be responsible for this difference, direct microwave coupling cannot be totally ruled out. However, our experiments essentially confirm the absence of resonance absorption of microwaves in HEW lysozyme at $v=8 \mathrm{GHz}$ up to power densities of about $0.3 \mathrm{~W} \mathrm{~mm}^{-2}$. Owing to the presence of water in globular proteins, intramolecular vibrations at microwave frequencies should be strongly damped and any remaining vibrational resonance should be extremely broad. The absence of resonance absorption of microwave radiation observed here in lysozyme, a representative for globular proteins, could thus be valid for many proteins in the whole microwave-frequency region.

We thank K. Achterhold, K. Dransfeld, S. Hayward, F. Parak and W. F. van Gunsteren for helpful discussions. This work was funded by the Optik-Zentrum Konstanz.

\section{References}

Adair, R. K. (2002). Biophys. J. 82, 1147-1152.

Bismuto, E., Mancinelli, F., d'Ambrosio, G. \& Massa, R. (2003). Eur. Biophys. J. 32, 628-634.

Bohr, H. \& Bohr, J. (2000). Phys. Rev. E, 61, 4310-4314.

Collaborative Computational Project, Number 4 (1994). Acta Cryst. D50, 760-763.

Dachwitz, E., Parak, F. \& Stockhausen, M. (1989). Ber. Bunsenges. Phys. Chem. 93, 1454-1458.

Dobrianov, I., Kriminski, S., Caylor, C. L., Lemay, S. G., Kimmer, C., Kisselev, A., Finkelstein, K. D. \& Thorne, R. E. (2001). Acta Cryst. D57, 61-68.

Fröhlich, H. (1968). Int. J. Quantum Chem. 2, 641-649.

Goldstein, L. S., Kheifets, L., van Deventer, E. \& Repacholi, M. (2003). Radiat. Res. 159, 275-276.

Gos, P., Eicher, B., Kohli, J. \& Heyer, W.-D. (1997). Bioelectromagnetism, 18, 142-155.

Harvey, S. C. \& Hoekstra, P. (1972). J. Phys. Chem. 76, 2987-94.

Hushcha, T., Peytcheva, A. \& Kaatze, U. (2002). J. Phys. Cond. Matter, 14, 9461-9464.

Joti, Y., Nakasako, M., Kidera, A. \& Go, N. (2002). Acta Cryst. D58, $1421-1432$.

Kabsch, W. (2001a). International Tables for Crystallography, Vol. F, edited by M. G. Rossmann \& E. Arnold, pp. 218-225. Dordrecht: Kluwer.

Kabsch, W. (2001b). International Tables for Crystallography, Vol. F, edited by M. G. Rossmann \& E. Arnold, pp. 730-734. Dordrecht: Kluwer.

Kiefersauer, R., Than, M. E., Dobbek, H., Gremer, L., Melero, M., Strobl, S., Dias, J. M., Soulimane, T. \& Huber, R. (2000). J. Appl. Cryst. 33, 1223-1230.

Kundi, M. (2003). Radiat. Res. 159, 274-278.

La Cara, F., D’Auria, S., Scarfi, M. R., Zeni, O., Massa, R., d'Ambrosio, G., Franceschetti, G., De Rosa, M. \& Rossi, M. (1999). Protein Pept. Lett. 6, 155-162.

Lerchl, A. (2003). Radiat. Res. 159, 276.

Lerina, I. V., Kovalenko, V. A., Belous, A. M., Rozanova, Y. D., Leonov, B. N. \& Morozova, T. F. (1997). Biophysics, 42, 1043-1047.

Polk, C. \& Postow, E. (1996). Editors. Handbook of Biological Effects of Electromagnetic Fields, 2nd ed. Boca Raton: CRC Press.

Pomerai, D. I. de, Smith, B., Dawe, A., North, K., Smith, T., Archer, D. B., Duce, I. R., Jones, D. \& Candido, P. M. (2003). FEBS Lett. 543, 93-97.

Porcelli, M., Cacciapuoti, G., Fusco, S., Massa, R., d'Ambrosio, G., Bertoldo, C., De Rosa, M. \& Zappia, V. (1997). FEBS Lett. 402, 102-106.

Postow, E. \& Swicord, M. L. (1996). Handbook of Biological Effects of Electromagnetic Fields, edited by C. Polk \& E. Postow, pp. 535580. Boca Raton: CRC Press.

Utteridge, T. D., Gebski, V., Finnie, J. W., Vernon-Roberts, B. \& Kuchel, T. R. (2002). Radiat. Res. 158, 357-364.

Utteridge, T. D., Gebski, V., Finnie, J. W., Vernon-Roberts, B. \& Kuchel, T. R. (2003). Radiat. Res. 159, 276-278.

Vaney, M. C., Maignan, S., Riès-Kautt, M. \& Ducruix, A. (1996). Acta Cryst. D52, 505-517.

Weissenborn, R., Reinhardt, T., Hansen, V., Maret, G. \& Gisler, T. (2004). Rev. Sci. Instrum. 75, 5253-5256. 